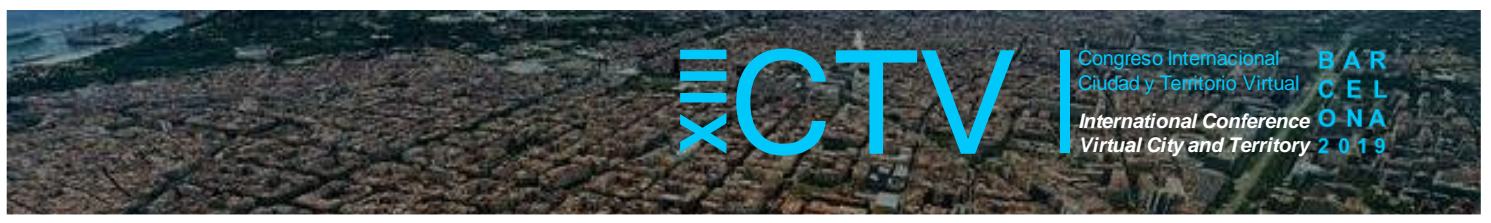

\title{
DERECHO A LA MOVILIDAD Y RUTAS DE RESILIENCIA. REUTILIZACIÓN Y RELANZAMIENTO DE FERROCARRILES SECUNDARIOS PARA LA REGENERACIÓN DE TERRITORIOS FRÁGILES EN ITALIA Y ESPAÑA
}

\author{
Amato, Chiara ${ }^{1 *}$; Ravagnan, Chiara ${ }^{2}$; Rossi, Francesca ${ }^{3}$ y de Ureña, José Maria ${ }^{4}$ \\ Remisión inicial: 2019-06-11; Remisión definitiva: 2019-10-17; Publicación: 2019-12-21
}

Citación: Amato, C. et al. (2019). Derecho a la movilidad y rutas de resilencias. Reutilización y relanzamiento de ferrocarriles secundarios para la regeneración de territorios frágiles en Italia y España. En XIII CTV 2019 Proceedings: XIII International Conference on Virtual City and Territory: "Challenges and paradigms of the contemporary city": UPC, Barcelona, October 2-4, 2019. Barcelona: CPSV, 2019, p. 8527. E-ISSN 2604-6512. DOI http://dx.doi.org/10.5821/ctv.8527

\section{Resumen}

Los procesos de metropolización del territorio impulsan los fenómenos de polarización espacial que determina un estado creciente de fragilidad económica y social de las "inner peripheries" conectado a problemas infraestructurales y medioambientales. En este contexto, el debate científico y las agendas políticas destacan la centralidad de los temas relacionados con la movilidad, que rediseñan las zonas rápidas y las zonas lentas de los países, el acceso a los servicios y el potencial de desarrollo sostenible, alimentando o debilitando la sangre vital del territorio, los flujos de personas y bienes. El Derecho a la Movilidad, finalizado a garantizar conexiones y accesibilidad, constituye una parte fundamental de una estrategia de regeneración dirigida a asegurar un nuevo welfare urbano, en el marco de las rutas de resiliencia para los territorios frágiles.

La reflexión ilustrada en esta ponencia es parte de la actividad de investigación que el grupo de académicos de las Universidades Sapienza, Roma Tre, UCLM y UPC están tratando sobre el tema de la regeneración urbana y territorial, poniendo en el centro el tema de las relaciones que existen entre urbanismo, infraestructuras para la movilidad y regeneración territorial, destacando los retos que plantea la presencia de redes ferroviarias en desmantelamiento.

Específicamente, la investigación intitulada Rutas de resiliencia. Reutilización y relanzamiento de ferrocarriles menores para la regeneración de los territorios frágiles. Experiencias en Italia y España aborda la reactivación de líneas ferroviarias menores en desuso, a través de un enfoque integrado, desde paisajes del descarte a ejes estructurantes de reequilibrio socio-económico y medioambiental, así como de reconfiguración morfólogica y de valorización paisajística.

La propuesta, a partir de la contextualización de las dinámicas en curso en Italia y España, como la marginalización de las "aree interne" y de la "España vacía", persigue el objetivo de identificar nuevas referencias metodológicas y operativas, a través de la definición de enfoques para la reactivación y reutilización de redes ferroviarias menores, en línea con las políticas y los programas orientados a responder a los procesos de desmantelamiento infraestructural.

La investigación adopta tres perspectivas consolidadas en el marco del debate urbanístico para analizar los fenómenos de fragilización y las experiencias de planes y proyectos, en coherencia con las políticas y los programas:

una perspectiva "estructural", que identifica el relanzamiento de los ferrocarriles como oportunidad para la revitalización socioeconómica de áreas marginales, a partir de una reactivación del servicio público, desde el "derecho a la movilidad";

una perspectiva "morfológica y paisajística", que centra la atención en la relación osmótica entre infraestructura y paisaje, a partir del reconocimiento de la forma como una herramienta cognitiva y de planificación para interpretar la identidad de los lugares y el disfrute como elemento indispensable para gobernar la percepción del territorio;

una perspectiva "ecológica y medioambiental", que considera la regeneración de la red ferroviaria como una oportunidad para el desarrollo sostenible, que sugiere la transformación en redes integradas verdes, portadoras de nuevas formas de movilidad sostenible y valores ecológicos colectivos.

En este marco, a partir de un proceso inductivo e iterativo de investigación, el documento sintetiza los primeros resultados de la investigación que subrayan la necesidad de nuevas categorías de planificación integrada e interescalar para la planificación de redes de infraestructura: corredores de movilidad rápida, ferrocarriles turísticos, vías verdes, nuevos criterios operativos que identifican los trazados como "figuras de regeneración" que ponen en sinergia herramientas ordinarias y extraordinarias, recursos públicos y privados multinivel, escenarios a largo, medio y corto plazo, nuevas formas de gestión de redes y de servicios internacionales y multinivel.

\footnotetext{
1 Dipartimento PDTA - Sapienza Università di Roma, https://orcid.org/0000-0002-7089-5538; ${ }^{2}$ Dipartimento PDTA, https://orcid.org/0000-0002-6036-1313; 3 Dipartimento PDTA, https://orcid.org/0000-0003-3057-6070; 4 UCLM, https://orcid.org/0000-0002-0404-1454. * Correo de contacto: chiara.ravagnan@uniroma1.it
} 


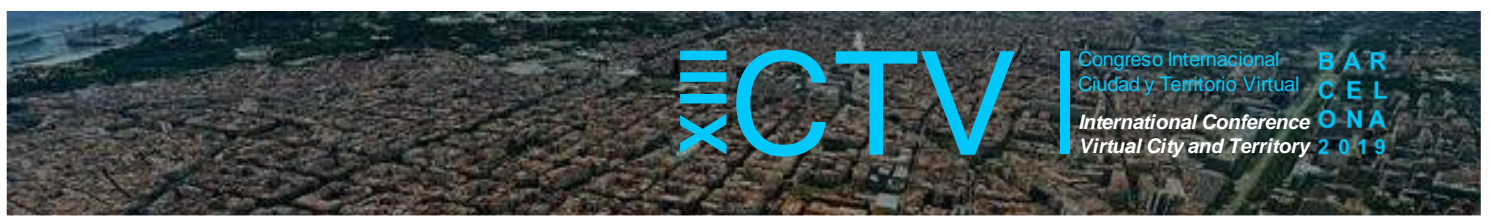

\section{Abstract}

The processes of metropolization of the territory foster the phenomena of spatial polarization that determines a growing state of economic and social fragility of the "inner peripheries" connected to infrastructure and environmental problems. In this context, the scientific debate and political agendas highlight the centrality of mobility, which redesign the "fast and slow areas" of the countries, the access to services and the potential for sustainable development, feeding or weakening the vital lymph of the territory, the flows of people and goods. The Right to Mobility, aimed to guarantee connections and accessibility, constitutes a fundamental part of a regeneration strategy to ensure a new urban welfare, within the framework of the resilience tracks for fragile territories.

In this context, the reflexion illustrated in this document is part of the research activity that the group of academics of the Universities La Sapienza, Roma Tre, UCLM and UPC are developing in the framework of urban and territorial regeneration issues, deepening the relationships between urban planning mobility, infrastructures and territorial regeneration and highlighting the challenges fostered by the presence of dismissed railways.

The research entitled "Resilience routes. Reuse and relaunch of minor railroads for the regeneration of fragile territories. Experiences in Italy and Spain" address the reactivation of underutilized or disused minor railway lines, through an integrated approach, moving from "landscapes of waste" to structuring axes of socio-economic and environmental rebalancing, as well as morphological reconfiguration and landscape valorization.

The proposal, based on the contextualization of the on-going dynamics in Italy and Spain, such as the marginalization of the "aree interne" and the "España vacía", aims to identify new methodological and operational references, through the definition of guidelines for the reactivation and reuse of minor railway networks, in line with policies and programs aimed at responding to divestment processes of infrastructures.

The research adopts three consolidated perspectives within the framework of the urban debate to analyze the phenomena of "fragilization" and cases of plans and projects, in consistence with the policies and programs:

a "structural" perspective, which identifies the regeneration of the railway network as an opportunity for the socioeconomic revitalization of marginal areas, based on a reactivation of the public service towards a "right to mobility";

a "morphological and landscape" perspective, based on the deepening of the form as a cognitive and planning tool to interpret the identity of places and enjoyment as an indispensable element to govern the perception of the territory, which focuses on the osmotic relationship between infrastructure and landscape;

an "ecological and environmental" perspective, which considers the regeneration of railways as an opportunity for sustainable development, which suggests the transformation into integrated green networks fostering new forms of slow mobility and collective ecological values.

In this framework, moving within an inductive and iterative research process, the document summarizes the first results of the research that underline the need for new integrated and intercalar planning categories for infrastructure networks: rapid mobility corridors, tourist railroads, green ways, new operational criteria that identify the roads as "regeneration figures" that put in synergy ordinary and extraordinary tools, multilevel public and private resources, long, medium and short term scenarios, as well as new international and multilevel forms of network and services management.

Palabras Clave: resiliencia; movilidad; sostenibilidad; ferrocarriles

Key words: resilience; mobility; sustainability; railways

\section{Territorios frágiles, regeneración y derecho a la movilidad}

En los últimos años, las agendas nacionales e internacionales se han centrado en el lado más desconcertante de la "metropolización del territorio", materializándose en los fenómenos de marginación social, empobrecimiento progresivo de los ecosistemas históricos, ambientales y productivos que ayudaron a construir la riqueza y la belleza de Europa. Hoy en día, estos parecen estar debilitados por su sangre vital, los flujos de personas y bienes que disminuyen en un círculo vicioso de desequilibrio socioeconómico, despoblación, desintegración de la infraestructura y exclusión social (Ravagnan, 2019).

Como se desprende de las actividades de investigación del programa Espon - Profecy (AAVV, 2017), en Europa están surgiendo situaciones de polarización y congestión de los grandes centros urbanos $\mathrm{y}$, al otro lado, de deterioro socioeconómico de vastas áreas geográficas conectadas a estructuras morfológicas, funcionales, demográficas específicas y a la 


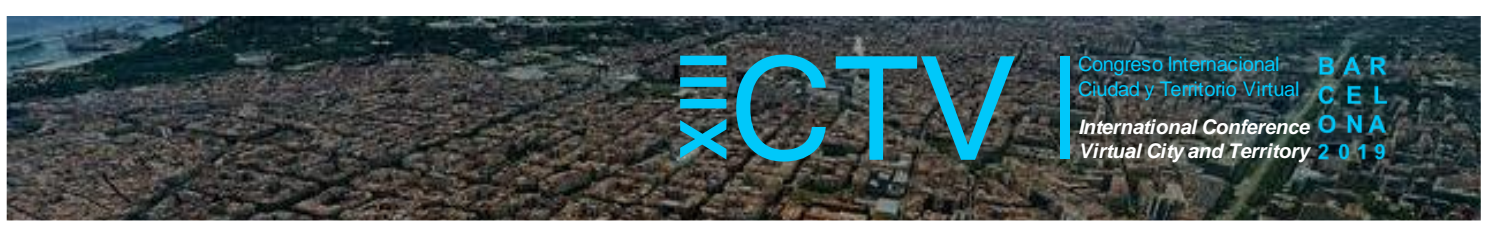

considerable distancia de las principales ciudades y de los principales centros de actividad económica y, en consecuencia, de servicios de interés general: las "inner peripheries". La comparación de los programas nacionales de investigación de los países europeos dibuja diferentes aspectos del equilibrio territorial entre áreas costeras y áreas de montaña, áreas congestionadas y áreas abandonadas, a partir del cual es posible delinear similitudes entre los activos de las zonas de la España vacía (Del Molino, 2016) y de las Aree interne italianas (SNAI 2014).

En la clasificación reciente de las "Aree interne" italianas, iniciada en 2012 por el entonces Ministero della Coesione Territoriale, se definen como un conjunto de pequeñas ciudades, pueblos, asentamientos de montaña, con niveles variables de periferia, áreas sustancialmente distantes de los centros de suministro de servicios, y caracterizadas por los procesos de degradación, despoblación y aislamiento.

Este aislamiento es el resultado de fenómenos culturales, como el boom de la década de 1960 en el transporte por carretera y la marginación del transporte público a una visión anacrónica y no muy moderna de la movilidad (Cerasoli, 2015), y políticos, a partir de las decisiones que impulsan transporte individual, liberalización ferroviaria y construcción de la Alta Velocidad. La AV aumenta una "geografía del poder" basada en territorios de alta y baja rentabilidad, aquellos atendidos por la red principal y por la AV, que refuerzan la conexión entre los núcleos más importantes y aquellos caracterizados por el efecto túnel, es decir la falta de estaciones en los espacios intermedios (de Ureña, 2012).

Estas dinámicas han producido serios costos sociales, ecológicos, ambientales y urbanos (desigualdades sociales, consumo de suelo, contaminación del aire, fragmentación urbana, disminución de la calidad de vida, reducción de los lugares de relación), que hoy recuerdan la necesidad de una estrategia que se enfoque hacia el transporte público, particularmente al ferrocarril, en su característica de red capilar, de medio sostenible de movimiento y de recurso para el reequilibrio territorial, dejando la lógica puramente de transporte para considerar las grandes infraestructuras como obras territoriales (Dematteis, 2001), un capital social fijo y, por lo tanto, un lugar de recomposición entre ciudadanos y territorio.

La situación de crisis multisectorial (socio-economica y medioambiental) que está afectando a las áreas más frágiles y marginales de países como Italia y España, y, en paralelo, las políticas y programas de movilidad de ferrocarriles, sugieren la importancia de reconocer el Derecho a la movilidad como un derecho primario de ciudadanía, no solo para los suburbios de los grandes centros urbanos - en el que se concentra el debate científico - pero sobre todo para las áreas marginales. En una "sociedad líquida", globalizada, basada en conexiones y velocidad de interacción, las infraestructuras para la movilidad sostenible constituyen la armadura territorial para la resiliencia de los territorios, ejes estratégicos de revitalización, capaces de definir los caminos rápidos y lentos, y para reactivar economías, dirigir flujos y redescubrir territorios.

El recurso para hacer esto es un capital social fijo que puede definirse como "un activo sin el cual las actividades productivas primarias, secundarias y terciarias no pueden funcionar (...) lo esencial del concepto puede restringirse al transporte y la energía" (Hirchmann, 1958).

En Italia, el patrimonio ferroviario abandonado consiste en alrededor de $7.500 \mathrm{~km}$, entre líneas en desuso y subutilizadas, mientras que en España es de unos 5.500 km (Cfr. Tabla 1). 


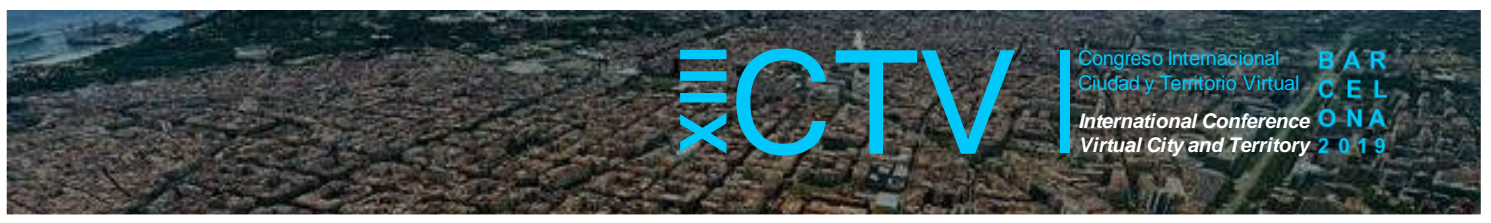

Tabla 1. Datos sobre territorios y ferrocarriles italianos y españoles

\begin{tabular}{|c|c|c|}
\hline & Italia & España \\
\hline Superficie tot & $302073 \mathrm{~km}^{2 \circ}$ & $504645 \mathrm{~km}^{2 \star}$ \\
\hline Población tot & $60.376 .408 \mathrm{ab}^{\circ}$ & $47.198 .000 \mathrm{ab}^{*}$ \\
\hline Densidad tot & $199,82 \mathrm{ab} \cdot / \mathrm{km}^{2} \circ$ & $92 \mathrm{ab} . / \mathrm{km}^{2 \star}$ \\
\hline Sup. áreas urbanas & $121.958 \mathrm{~km}^{2 \circ 0}$ & $236.562 \mathrm{~km}^{2 \star \star}$ \\
\hline \% sup. áreas urbanas & $40 \%$ & $47 \%$ \\
\hline Población áreas urbanas & $46.153 .200 \mathrm{ab}^{\circ \circ}$ & $39.418 .215 a b^{\star *}$ \\
\hline \% población áreas urbanas & $\mathbf{7 7 , 7} \%$ & $84,89 \%$ \\
\hline Sup. áreas internas & $180.115 \mathrm{~km}^{2 \circ 0}$ & $268.083 \mathrm{~km}^{2 \star \star}$ \\
\hline$\%$ sup. áreas interne & $60 \%$ & $53 \%$ \\
\hline Población áreas internas & $13.280 .544 \mathrm{ab}^{\circ 0}$ & $7.133 .237 a b^{\star \star}$ \\
\hline \% población áreas internas & $22,3 \%$ & $15,11 \%$ \\
\hline Ferrocarriles tot & $26500 \mathrm{~km}^{\circ 00}$ & 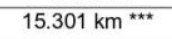 \\
\hline $\begin{array}{l}\text { Ferrocarriles abandonados } \\
\text { (en desuso y suspendido) }\end{array}$ & $7500 \mathrm{~km}^{\circ 00}$ & $5.476 \mathrm{~km}^{\star \star \star \star \star}$ \\
\hline
\end{tabular}

ISTAT - Istat Istituto di Statistica 2019 Strategia Nazionale Aree Interne con datos ISTAT. Por áreas urbanas entendemos aquellas clasificadas como Polo, Polo intermedio y Cintura. Por áreas internas entendemos aquellas clasificadas como Intermedio, Periferico y Utraperiferico Database Ferrovieabbandonate.it con datos Ferrovie dello Stato como a lineas deconcesion. desuso nos referimos tanto a lineas $F$

Instituto Nacional de Estadistica 2018. Para el cálculo de las áreas internas se tiene en cuenta la subdivisión de Espana vacia Sergio de Molino (Regiones áreas internas de Aragona, Castilla - La Mancha, Castilla y Leon, Extremadura, La Riaoja - Regiones áreas urbanas: Andaluciá, Principado de Asturias, Illes Balears, Canarias, Cantabria, Cataluña, Comunitat Valenciana, Galicia, Comunidad de Madrid, Región de Murcia, Comunidad Foral Navarra, Pais Vascol Ministerio de Fomento, Goberno de España (ajos ferrocarriles Ferroviarias https://www.elmundo.es/economia/2017/09/04/59ac4ad5e2704e1 1478b45a4.html

Fuente: C. Amato, 2019

Estas líneas abandonadas, con el cese del servicio han traído consigo fragmentos de territorio, fragmentos de economías, restos de vida, como raíces arrancadas del suelo (Amato et al., 2019), en un círculo vicioso de abandono y despoblación que es necesario revertir dando nueva vida a estas "ramas secas".

\section{Rutas de resiliencia. Objetivos y metodologia de investigación}

Los frentes más avanzados del debate sobre la regeneración sugieren la oportunidad de contrastar la marginalidad y el abandono de las "inner peripheries", poniendo en el centro de las estrategias territoriales las opciones vinculadas al fortalecimiento, la reutilización y la reactivación de las redes infraestructuales (Carta, Ronsivalle, 2015; Cerasoli, Mattarocci, 2018). Estas redes se configuran como componentes estratégicos, "matrices de regeneración" capaces de contrastar el debilitamiento progresivo de la "sangre vital" de los territorios sujetos al abandono y la despoblación y de experimentar "rutas de resiliencia" a los cambios sociales, económicos y medioambientales (Ravagnan, 2019). Recuperar a los restos de infraestructura "desde fondo hasta figura" para la construcción de escenarios de regeneración es una operación compleja pero indispensable en lo que concierne a la habitabilidad y la seguridad de los territorios. Aspectos que no dependen de la calidad técnica de algunas intervenciones puntuales y sectoriales, sino de "la calidad general y la escala múltiple de las redes de relación: física, cultural, económica, social" (Di Venosa et al., 2017).

En este contexto, la investigación internacional "Rutas de resiliencia. La reutilización y el relanzamiento de los ferrocarriles secundarios para la regeneración de territorios frágiles. Experiencias en Italia y España", que el grupo de investigacion está llevando a cabo, se fija como objetivo recomponer las referencias metodológicas y operativas para el relanzamiento y la reutilización de líneas ferroviarias en el contexto de estrategias generales para la regeneración de territorios frágiles. Líneas de intervención, por lo tanto, en el marco de un enfoque integrado que contempla diferentes perspectivas disciplinares y diferentes políticas, portadoras de nuevas categorías de proyectos, criterios operativos, formas de gestión y canales de financiación.

Un enfoque que asegura, en los procesos de intervención sobre las infraestructuras, la activación de acciones de reorganización funcional y revitalización socioeconómica, mejoramiento morfológico-paisajístico de los contextos territoriales y regeneración ambiental. 


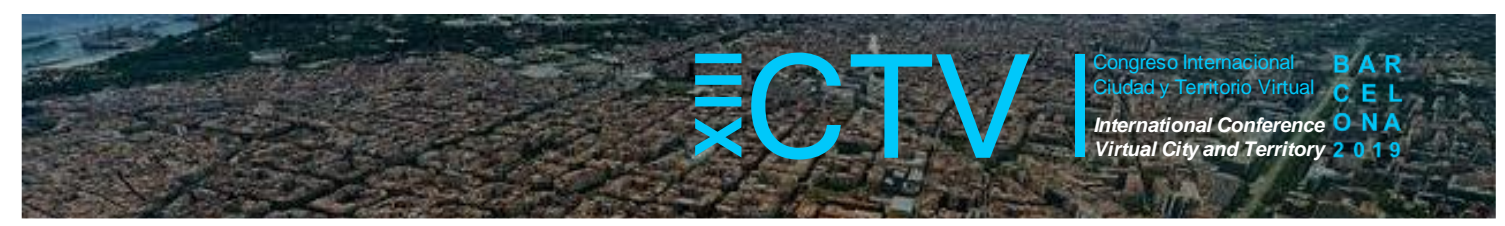

Un enfoque en el que las vías ferroviarias se configuran como ejes estructurantes de reequilibrio territorial, como redes de lugares de identidad, como infraestructuras integradas (gris y verdes) portadoras de nuevas formas de movilidad sostenible y nuevos valores colectivos orientados al medioambiente y a la memoria. Un enfoque que parte de la conciencia de la profunda interrelación entre las diferentes formas de "fragilidad": la fragilidad social vinculada a la contracción de los derechos de ciudadanía y la disminución de la calidad de vida de las personas, al origen de los fenómenos de despoblación, la fragilidad económica que engendra un círculo vicioso de empobrecimiento del patrimonio público y privado, a la fragilidad ecológico-ambiental que alimenta el abandono y determina la escasa seguridad de los contextos "sensibles". Este enfoque asume el concepto de "resiliencia" como referencia contra el abandono y la despoblación de "áreas internas" (Italia), "vacías" (España) y recuerda el carácter proactivo del dinamismo y la adaptación a los retos de adaptación a los cambios ambientales y socioculturales. En esta dirección, siguiendo la fase de contextualización de los fenómenos, la investigación adopta una metodología inductiva e iterativa, para reconstruir las referencias metodológicas y operativas a partir del examen del debate y las experiencias en curso (políticas y programas, planes y proyectos), favoreciendo algunos "paradigmas", puntos de vista, perspectivas complementarias útiles para el análisis de la experimentación (Ricci et al., 2018) articuladas instrumentalmente en:

- una primera perspectiva "estructural" que identifica las acciones en la red ferroviaria como oportunidades para la revitalización socioeconómica de las "inner peripheries" a partir de una recuperación del servicio público, contrastando los efectos que la liberalización ha tenido en el transporte ferroviario y de pérdida de la vocación pública del servicio y la distribución justa del "derecho a la movilidad" como derecho de la ciudadanía; en esta perspectiva, la investigación propone diferentes experiencias que recogen el papel fundamental del transporte público en las áreas cada vez menos accesibles y pobladas, para el mantenimiento de estilos de vida sostenibles y el acceso a lugares centrales, a los servicios básicos como educación, salud, cultura, coordinando los sistemas de trasporte público genérico con los sistemas de transporte sectoriales (sanitario, postal, educativo, etc.) para crear formas de movilidad más sostenible (Ruiz de la Riva et al., 2015);

- una segunda perspectiva "morfológica y paisajística" se centra en la relación osmótica entre infraestructura y paisaje, a partir del reconocimiento de la forma como una herramienta cognitiva y de diseño para captar la identidad de los lugares y la fructificación como elemento indispensable para gobernar la percepción del territorio. En este sentido, la dimensión paisajística de las infraestructuras ferroviarias constituye un tema emergente de reflexión que desarrolla el carácter de identidad de los "ferrocarriles turísticos", a través de líneas destinadas a la valorization del paisaje y del patrimonio abandonado - líneas, estaciones, puentes, viaductos y túneles, junto con la revitalización de los contextos territoriales.

- una tercera perspectiva "ecológica-ambiental" considera la regeneración de la red ferroviaria como un impulsor del desarrollo sostenible y de la regeneración medioambiental, sugiriendo la transformación hacia "vías verdes". Una perspectiva que intenta estructurar infraestructuras verdes, trayendo nuevas conexiones entre áreas de alta naturalidad, nuevas formas de movilidad y usos ecológicos en contextos frágiles y nuevas economías circulares y verdes conectadas a valores colectivos orientados al cuidado y al respeto por el medio ambiente. 


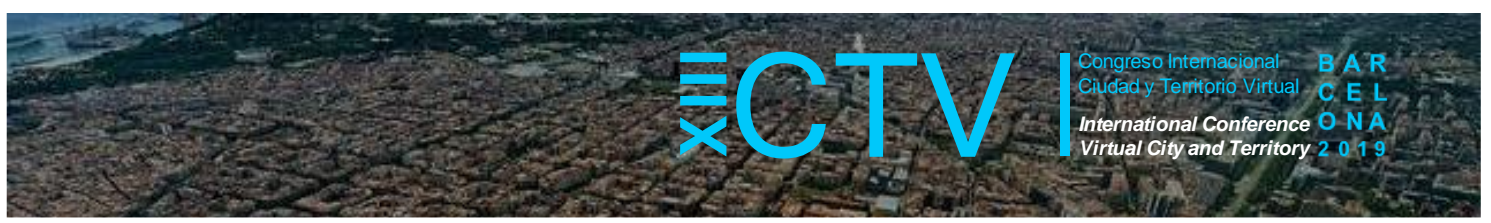

En coherencia con estas perspectivas, se ilustran las políticas y programas centrados en territorios frágiles y en el relanzamiento y reutilización de líneas ferroviarias y, en relación con estos marcos político-legislativos, se analizan casos emblemáticos de planes y proyectos, en los que es posible destacar el papel principal atribuido al abandono de las líneas de ferrocarril en los procesos de "fragmentación" de los territorios y, al mismo tiempo, las oportunidades de regeneración derivadas de las intervenciones en infraestructuras, verdaderas "rutas de resiliencia", portadoras de nuevas economías, nuevos lugares de identidad y nuevos valores ecológicamente orientados.

\section{Italia y España. Políticas y programas nacionales y europeos entre revitalización y reutilización de líneas ferroviarias abandonadas}

En España, donde en el año 2018, el 61,5\% del total de municipios contaban con menos de 1.000 habitantes, la Federación Española de Municipios y Provincias (FEMP) ha afirmado en un "Documento de Acción de la Comisión de Despoblación" del año 2017 que la regresión demográfica se ha convertido en un problema nacional y como tal ha de responderse a él desde una perspectiva de gobernanza multinivel que, en la práctica, conlleva la implicación de todas las administraciones públicas en todos sus frentes de acción.

En este sentido la FEMP plantea el reequilibrio territorial y la lucha contra la despoblación como una inversión en cohesión social y territorial y en fortaleza y sostenibilidad del modelo económico y social, a partir de las infraestructuras, servicios básicos y comercios.

Al mismo tiempo, en Italia, dentro del Plan de Reforma Nacional 2014, se lanzó una Strategia Nazionale per le Aree Interne (SNAI), administrada por la Agenzia per la Coesione Territoriale, para relanzar el desarrollo y los servicios de estas áreas a través de fondos ordinarios de la Legge di stabilità $2015 \mathrm{y}$ fondos de la UE 2014-2020 (FEDER, FSE, FEADER, FEMP).

El objetivo es contrarrestar el declive demográfico de las "aree interne", tomando nota de las condiciones de marginación que éstas han sufrido y desplegando acciones para adaptar los servicios esenciales, salud, educación y movilidad, que hacen frente a las instancias de ciudadanía y los proyectos de desarrollo local, que mejoran el patrimonio natural y cultural de estas áreas.

Dentro de la SNAI, el tema de la movilidad es emblemático de la complejidad con la cual abordar la intervención en las "aree interne" y del potencial económico, social y medioambiental de las intervenciones, como se desprende de las Linee guida per gli interventi sulle aree progetto (Cfr. Tabla 2) que, a partir del reconocimiento del Derecho a la movilidad como un derecho de la ciudadanía, intentan responder a una necesidad de movilidad (ordinaria y turística).

En estos contextos, la presencia de las líneas ferroviarias abandonadas aparece como una oportunidad tanto para la reactivación del servicio ferroviario, a través de nuevos corredores para la movilidad ordinaria/general en ferrocarril, como para una perspectiva turística, a través del establecimiento de trenes históricos, así como para la conversion de los trazados en vías verdes (Amato, 2018). 


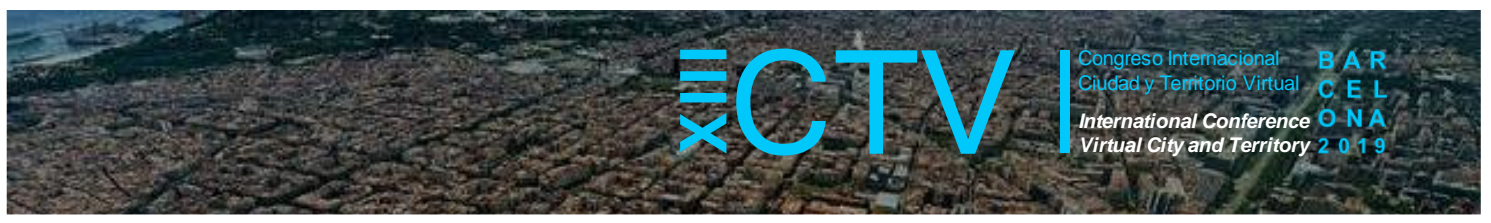

Tabla 2. Clasificación de las necesidades de movilidad expresadas en las "áree interne"

\begin{tabular}{|c|c|}
\hline Macro-fabbisogni & Fabbisogni specifici \\
\hline \multirow{2}{*}{$\begin{array}{l}\text { Pianificazione e } \\
\text { Programmazione dei } \\
\text { sistemi di trasporto }\end{array}$} & $\begin{array}{l}\text { Promuovere l'accessibilità dei luoghi, garantendo il diritto di mobilità delle } \\
\text { persone e delle opportunità di scambio dei beni sul territorio, attraverso una } \\
\text { visione integrata del sistema trasporto-territorio mediante l'adozione di strument } \\
\text { di pianificazione e programmazione dei trasporti di tipo "strategico" (scenario a } \\
10 \text { anni) }\end{array}$ \\
\hline & $\begin{array}{l}\text { Promuovere l'accessibilità ed il diritto di mobilità sul territorio dell'Area interna } \\
\text { con strumenti di pianificazione e programmazione dei trasporti di tipo "tattico" } \\
\text { (scenario a } 3 \text { anni) }\end{array}$ \\
\hline \multirow{4}{*}{$\begin{array}{l}\text { Potenziamento e } \\
\text { riqualificazione della } \\
\text { dotazione } \\
\text { trasportistica }\end{array}$} & $\begin{array}{l}\text { Incrementare l'accessibilità interna e esterna all'area attraverso l'adeguamento } \\
\text { delle infrastrutture di trasporto }\end{array}$ \\
\hline & $\begin{array}{l}\text { Promuovere l'accessibilità e l'attrattività del TPL con l'adeguamento dei nodi di } \\
\text { accesso, l'infomobilità ed il rinnovo della flotta }\end{array}$ \\
\hline & $\begin{array}{l}\text { Promuovere l'accessibilità degli attrattori turistici del territorio privilegiando le } \\
\text { opportunità di una "fruizione sostenibile" delle risorse ambientali e culturali }\end{array}$ \\
\hline & $\begin{array}{l}\text { Sostenere il diritto di mobilità delle persone a capacità motoria ridotta a causa di } \\
\text { condizioni fisiche e/o socio-economiche svantaggiate }\end{array}$ \\
\hline \multirow{5}{*}{$\begin{array}{l}\text { Sviluppo dei servizi } \\
\text { di trasporto }\end{array}$} & $\begin{array}{l}\text { Facilitare l'accessibilità ai poli funzionali (scuola, lavoro) della mobilità } \\
\text { "sistematica" dei residenti }\end{array}$ \\
\hline & $\begin{array}{l}\text { Facilitare l'accessibilità ai poli funzionali (sanitari, culturali, sportivi, turistici } \\
\text { ecc.) della mobilità "non sistematica" di residenti e non residenti (turisti, } \\
\text { visitatori in genere) }\end{array}$ \\
\hline & Agevolare e sostenere il diritto di mobilità degli utenti a capacità motoria ridotta \\
\hline & $\begin{array}{l}\text { Promuovere la coesione territoriale dell'Area interna e l'accessibilità da e verso } \\
\text { poli esterni attraverso lo sviluppo di servizi di trasporti, anche a favore dei turisti }\end{array}$ \\
\hline & $\begin{array}{l}\text { Favorire le opportunità di fruizione sostenibile delle Aree interne per i residenti } \\
\text { i turisti incentivando servizi di modalità "lenta o dolce" sulle reti pedonali e le } \\
\text { greenways }\end{array}$ \\
\hline
\end{tabular}

Fuente: Snai-Mit-Isfort http://territori.formez.it/sites/all/files/areeinterne lineeguidatrasporti.pdf

En particular, algunas experiencias demuestran que las Regiones pueden tomar protagonismo en la reorganización y el relanzamiento definiendo algunas líneas prioritarias para ser mantenidas como servicio público, junto con los gobiernos nacionales que pueden financiar el déficit de funcionamiento de estas líneas. De hecho, si en áreas urbanas, la reutilización de líneas ferroviarias en desuso se traduce principalmente en nuevas oportunidades para movilidad alternativa (eléctrica, autónoma, etc.), en áreas internas (vacías) el uso de estos recursos latentes puede apoyar estrategias de desarrollo local y regeneración más complejas.

El carácter interescalar de las estrategias de regeneración de los territorios frágiles y marginales en relación con la revitalización del servicio ferroviario sólo puede adoptar un enfoque multiescalar hacia una posible integración con los escenarios europeos y nacionales, aprovechando las oportunidades indispensables otorgadas por los programas de financiación.

La UE representa el contexto donde se describen las grandes estrategias de planificación territorial, las prioridades de acción y los programas de financiación relevantes para los proyectos de infraestructuras. En particular, en 1992 se lanzó el proyecto TEN (Trans European Networks), que luego se actualizó con la red TEN-T definida por el Reglamento europeo $1315 / 2013$, destinado a crear una red de transporte europea interconectada e integrada, tanto geográficamente como para sistemas de transporte, con el objetivo de garantizar la continuidad de las rutas, crear nuevas redes (ferrocarriles de alta velocidad y autopistas), adaptar las redes existentes, armonizar la tecnología y hacer que los distintos segmentos sean interoperables.

De los nueve corredores que constituyen la columna vertebral de la red TEN-T, cuatro interesan a Italia, cruzándola de norte a sur y de oeste a este, el Báltico-Adriático, el Escandinavo-Mediterráneo, el Rin-Alpino, el Mediterráneo, mientras que España está atravesada por los corredores Mediterráneo y Atlántico, que la cruzan de este a oeste. La 


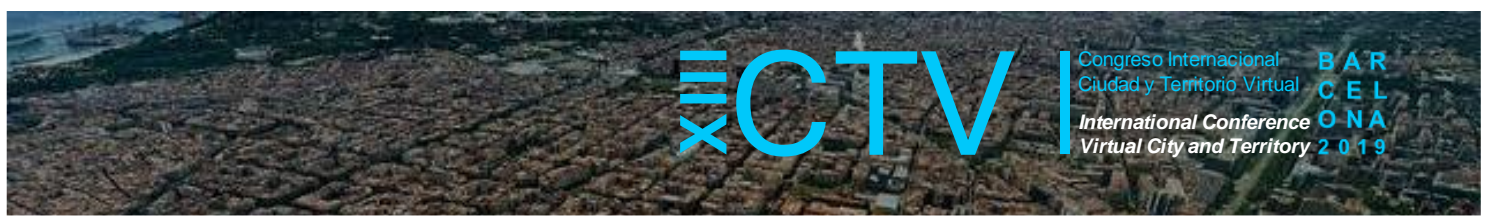

presencia de estos corredores estratégicos que atraviesan las fronteras nacionales ha tenido efectos en los corredores de alta velocidad, pero también en la reactivación de líneas abandonadas conectadas con estas, que se han convertido en prioridades en la perspectiva de la red y, por lo tanto, en el tema de los programas y fondos especiales europeos (cfr. § 4.1). En esto sentido, en España, un estudio llamado TREN 2020 plantea un grupo de propuestas para potenciar la oferta y la demanda del ferrocarril, a partir de un encargo de Comisiones Obreras, Greenpeace y WWF a la Asociación para la Promoción del Transporte Público (PTP), y tiene como principal objetivo optimizar la política de infraestructuras y servicios del Gobierno de España para potenciar aún más el ferrocarril. Se propone una nueva hoja de ruta basada en la combinación de planes de servicio (2013-2020) con planes de infraestructura (2013-2030). El 2020 da nombre a este proyecto al ser éste el plazo fijado por la Unión Europea para haber reducido un 20\% las emisiones de CO2 respecto a 1990.

Aquellas líneas abandonadas que cruzan territorios de valioso paisaje, con el establecimiento de servicios de trenes turísticos, incluso con vehículos históricos que han sido reestructurados, pueden constituir hoy, tambien, un gran recurso tanto en términos de mantenimiento de línea (tal vez pendiente de una reactivación del servicio de tren ordinario) tanto en términos de usabilidad turística de pequeños centros como de apoyo para la planificación intermunicipal de eventos e itinerarios.

Tabla 3. Acciones, políticas y casi estudios de investigación

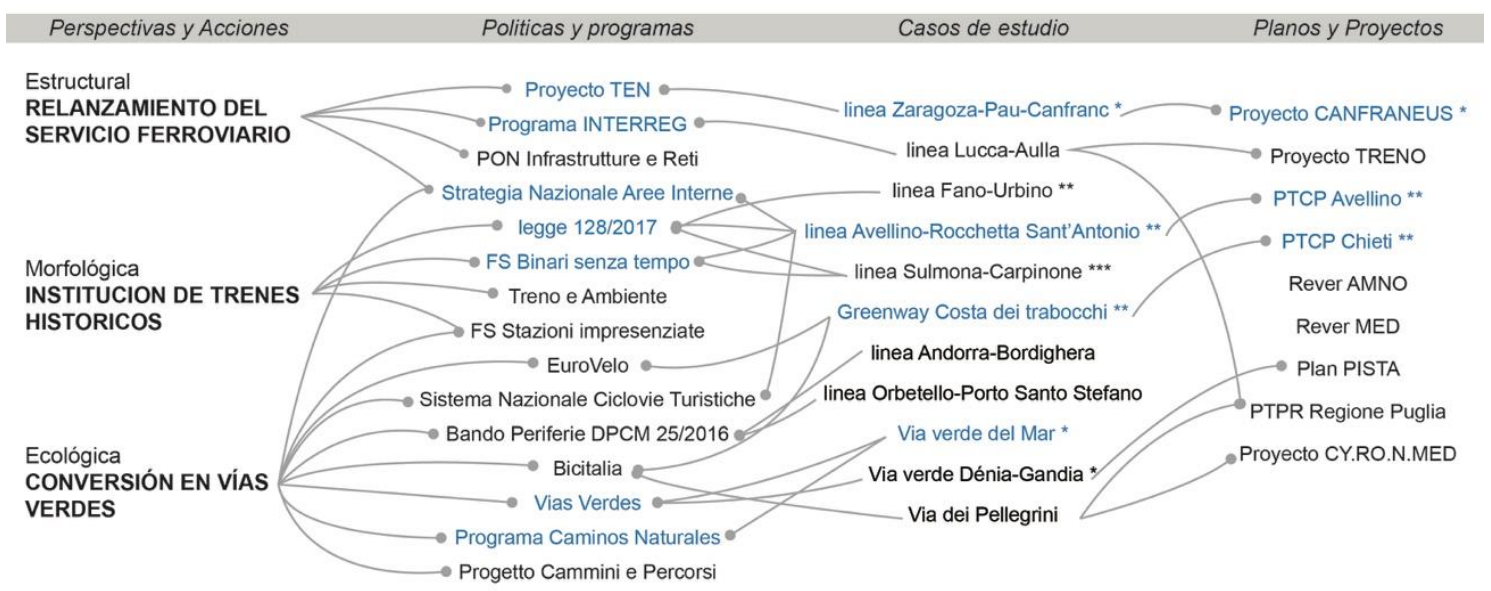

Fuente: C. Amato, 2019.

En Italia, en este sentido, desde 2014, la Fondazione FS italiane ha lanzado la iniciativa Binari senza tempo, que envolvió alrededor de $240 \mathrm{~km}$ de líneas sin servicio de transporte, que se han convertido en un museo dinámico que cruza los más lindos paisajes italianos, involucrando a unos 150.000 turistas cada año. Esta tendencia ha conducido en 2017, con la aprobación de la Ley n.128/17 "Disposizioni per l'istituzione di ferrovie turistiche mediante il reimpiego di linee in disuso 0 in corso di dismissione situate in aree di particolare pregio naturalistico 0 archeologico", al establecimiento de 18 ferrocarriles turísticos a través de la reutilización de líneas en desuso en áreas prestigiosas, también previendo la creación de nuevos espacios museísticos que, junto con la mejora de los vehículos ferroviarios históricos, permitirán enriquecer la propuesta turística del país, mejorar la movilidad sostenible y ofrecer nuevas e importantes oportunidades laborales. Como parte de las actividades de valorización de los 


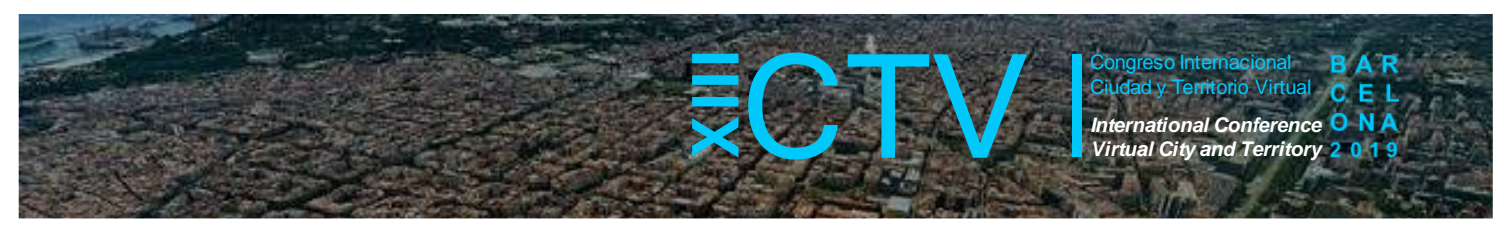

tramos ferroviarios afectados, serán involucradas las asociaciones y organizaciones de voluntarios que trabajan en el ferrocarril, el turismo, la cultura y el medio ambiente (cfr. § 4.2).

En España, la Fundación de los Ferrocarriles Españoles (FFE), constituida en 1985, junto a las principales empresas del sector público ferroviario español, tiene encomendada la recuperación y valorización del patrimonio histórico, cultural, científico y tecnológico ferroviario. La Fundación también recoge, como se puede leer en su estatuto, el fin de “...desarrollar y mantener el Plan de identificación, protección y divulgación del Patrimonio Histórico y Cultural Ferroviario" (Art. 7). La Fundación también desarrolla la plataforma de turismo ferroviario TouRail, un proyecto que persigue la promoción coordinada de todas las iniciativas españolas ligadas al patrimonio ferroviario, con el fin de generar hitos atractivos como estaciones monumentales, trazados, viaductos y museos a través de los trenes turísticos.

Con respecto a las estrategias para reutilizar líneas desmanteladas, a nivel europeo, además de la integración de la movilidad en bicicleta en muchos proyectos de transporte y líneas transeuropeas (TEN-T), se han elaborado proyectos para la creación de redes para la movilidad ciclo peatonal europea, como el proyecto EuroVelo, elaborado por la Federación Europea de Ciclistas por primera vez en 1997 que incluye, en 2013, 45,000 km ya utilizables, conectando las líneas ciclo peatonales existentes de los distintos estados miembros y planificando nuevas secciones para lograr el diseño de rutas accesibles y continuas (Cfr. § 5.1). La FFE en ESPANA coordina, a escala nacional, las diferentes iniciativas del Programa Vías Verdes, desarrollado desde 1993 por el anterior Ministerio de Obras Públicas, Transportes y Medio Ambiente y actualmente por el Ministerio de Agricultura, Alimentación y Medio Ambiente, en colaboración con Adif, Renfe y Feve. Un Programa en el que participan muy activamente las comunidades autónomas, diputaciones y ayuntamientos, así como grupos ciclistas, ecologistas y ciudadanos para desarrollar iniciativas de reutilización de los ferrocarriles en desuso, o que nunca llegaron a prestar servicio, con fines ecoturísticos (Cfr. § 5.2). Estas políticas se reflejan en casos de reutilización y relanzamiento de infraestructuras en Italia y España, que serán profundizadas en nuestra investigación (tabla 2). Algunos casos emblemáticos van a ser ilustrados en los apartados 4 y 5 .

\section{Experiencias de revitalización del servicio ferroviario}

\subsection{La línea Zaragoza-Canfranc-Pau}

En una perspectiva estructural de regeneración de las "inner peripheries", los contextos montañosos transfronterizos, marginales son casos emblemáticos "por excelencia", como el área de los Pirineos, teatro de la reactivación de la línea ferroviaria internacional ZaragozaCanfranc-Pau, que atraviesa la Comunidad Autónoma española de Aragón y la región francesa de Nueva Aquitania. Inaugurada en 1928 por el Rey de España y el Presidente de la República Francesa, representó una intervención importante para la red infraestructural transfronteriza, capaz de facilitar los intercambios entre España y Francia y el paso de viajeros por los Pirineos. La longitud total de la línea, de $307 \mathrm{~km}$, se divide en un tramo español de $214 \mathrm{~km}$, desde Zaragoza hasta Canfranc, y uno francés de $93 \mathrm{~km}$ entre Canfranc y Pau. La estación de Canfranc, completada junto con la línea en 1928, fue el principal punto de acceso a España desde Francia y hoy representa un lugar de memoria y calidad arquitectónica (que en la actualidad está reconvirtiéndose en un hotel junto con la creación de una nueva estación). El 


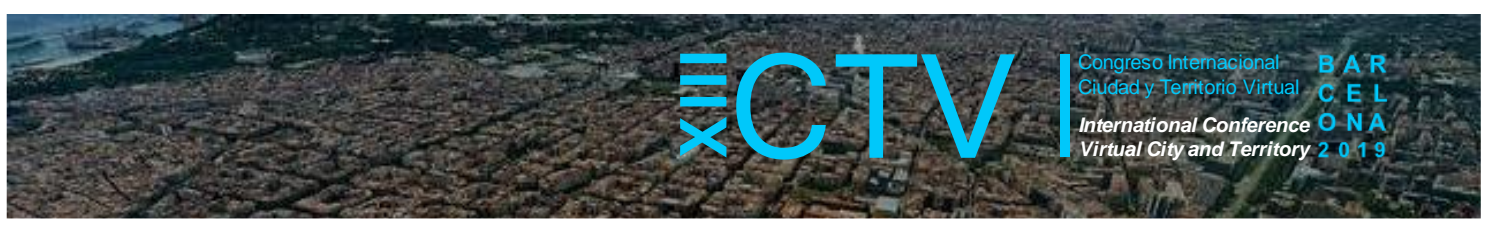

servicio fue interrumpido por tramos sucesivos desde la década de 1970 hasta la década de 1980, por un accidente y por la competitividad del transporte automóvil.

Además la característica transfronteriza ha influido en el destino de la línea, vinculándola con los eventos de la Guerra Civil Española y con las dinámicas políticas España-Francia, pero también ha significado que, a partir de 2000, la reapertura de la línea se convertiría en un objetivo transversal de organismos territoriales institucionales, Ministerios, Regiones, representantes del sector socioeconómico (Cámara de Comercio, Asociaciones Comerciales, Confederaciones de empresarios) y ciudadanos. La reapertura de la línea permite el transporte de mercancías entre las dos naciones a través del ferrocarril, que actualmente cubre solo el $3.2 \%$ del total contra el paso de unos veinte mil camiones al día, con las consiguientes cuestiones de seguridad, congestión, contaminación acústica y emisiones de $\mathrm{CO}^{2}$. Ademas, la línea tiene una característica estratégica ante una eventual independencia del País Vasco y/o Cataluña de España. El proyecto, apoyado a nivel europeo a través del FEDER para mejorar la accesibilidad de los Pirineos, lleva a cabo efectivamente el tercer paso entre los dos corredores prioritarios de la TEN-T, el Corredor Atlántico al oeste y el Corredor Mediterráneo al este. En este marco de referencia, dentro del Programa Operativo de Cooperación Territorial entre España-Francia-Andorra (POCTEFA INTERREG IV A, 2007-2013 y 2014-2020), el Gobierno de Aragón y el Consejo Regional de Aquitania han presentado el proyecto conjunto CANFRANEUS y CANFRANEUS II, destinado a restablecer el tráfico ferroviario en la línea.

\section{Figura 1. La linea Zaragona-Pau-Canfranc y la Red TEN}

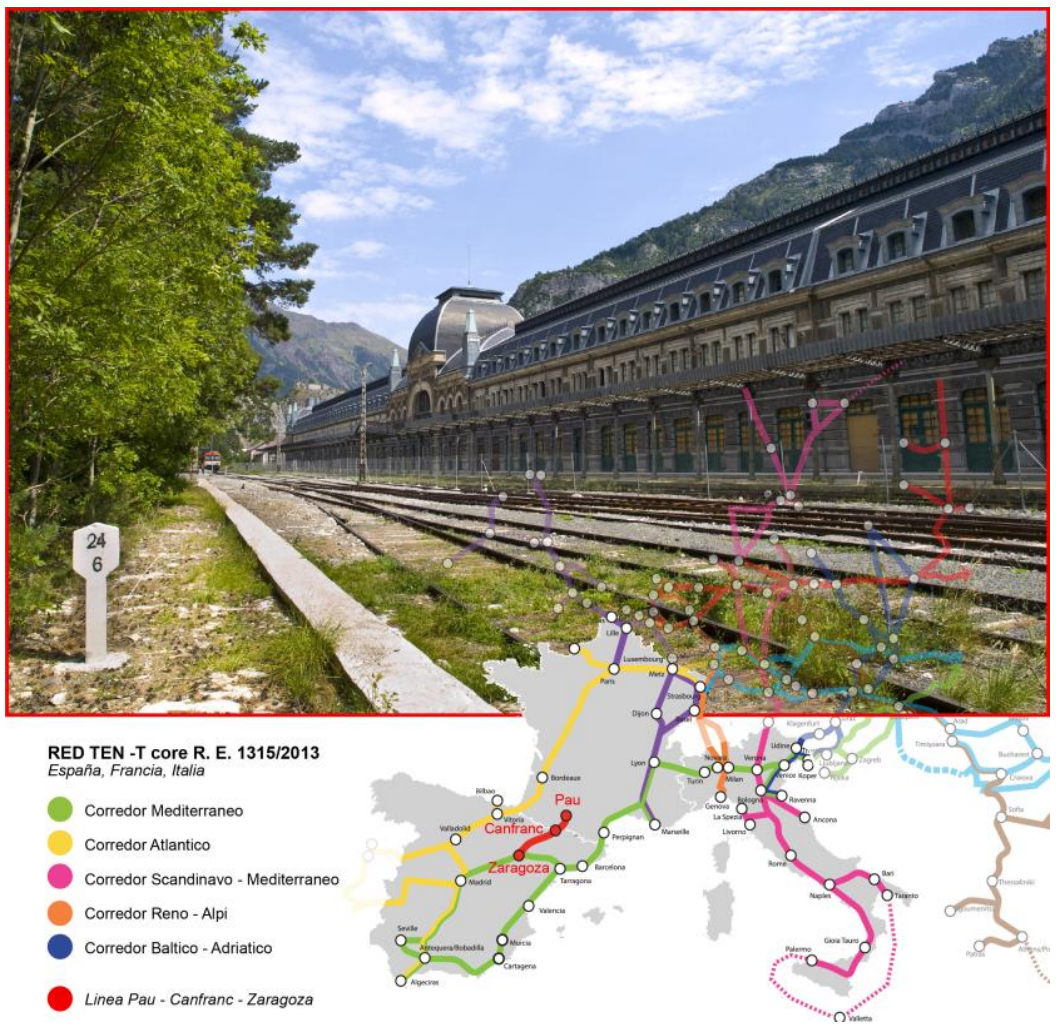

Fuente: C. Amato, 2019. Foto: iStock.com/Somatuscani

La reapertura en 2016, de un primer tramo en Francia entre Olorón y Bedous es, la expresión tangible del deseo de proceder a la reapertura de toda la línea. La intervención posterior, que prevé la restauración, por parte del gobierno español, del tramo Huesca - Canfranc, para 2020, 


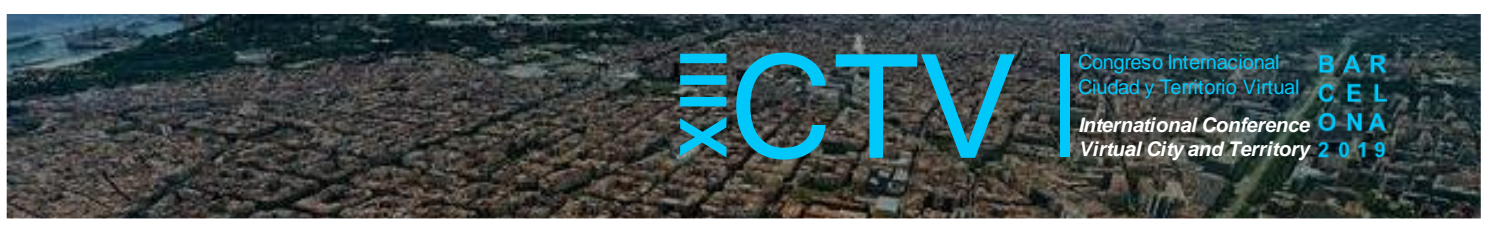

sancionada por un protocolo entre Aquitania y Aragón, concreta el primer objetivo del proyecto: mejorar el servicio de transporte dirigido a los viajeros y al mismo tiempo, fomentar el uso de la línea ferroviaria con fines comerciales. Por lo tanto, el caso de la reactivación de esta línea muestra una voluntad compartida por todos los niveles institucionales para estructurar una red de transporte integrada entre grandes corredores ferroviarios y líneas de conexión transversales, facilitando el desarrollo económico y turístico sostenible en áreas transfronterizas, como los peregrinos hasta Santiago de Compostela, y protegiendo los contextos más frágiles, como el de la Valle de Aspe, un importante corredor ecológico.

\subsection{La linea Avellino-Rocchetta Sant'Antonio}

Con respecto al relanzamiento del servicio ferroviario en una perspectiva morfológica, que se ocupa del tren en su conjunto y del paisaje atravesado, la línea Avellino - Rocchetta Sant'Antonio es uno de los ferrocarriles turísticos más prestigiosos enumerados en la Ley 128/2017. La linea de ferrocarril de $120 \mathrm{~km}$ cruza el territorio rico en historia, cultura y paisajes de Irpinia. El camino, muy tortuoso, cruza tres regiones, Campania, Basilicata y Puglia, a través de un territorio frágil y un paisaje caracterizado por pueblos casi abandonados, emblemáticos de la dinámica que afecta a las áreas internas. La línea, inaugurada en 1895, conectaba Avellino con la constelación de pequeños pueblos construidas a lo largo de los valles de los ríos Sábado, Calore y Ofanto, ejes de conexión antes del advenimiento del ferrocarril. Pero la ausencia de su inserción real en una red infraestructural y la falta de conexiones cruzadas con el contexto circundante, junto con el auge del automóvil y la construcción de la Carretera Nápoles-Bari, hizo progresivamente que se vaciase el área de influencia del ferrocarril.

Figura 2. La linea Avellino-Rocchetta-Sant'Antonio y las Ferrocarriles Turisticas

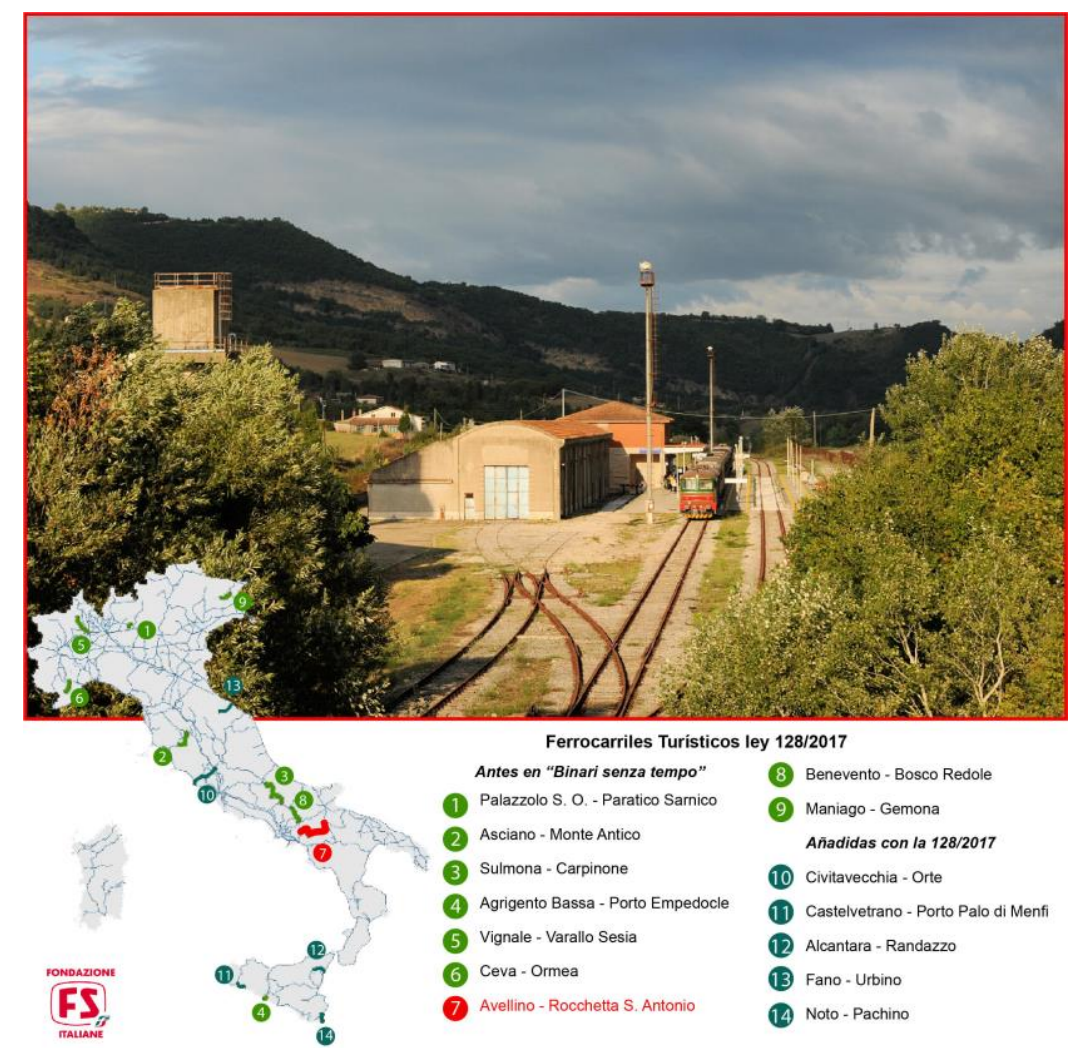

Fuente: C. Amato, 2019. Foto: Andrea Terrevoli. 


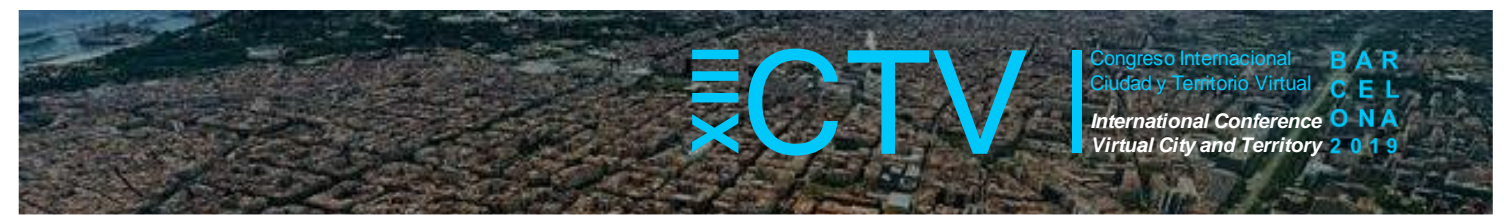

El éxodo de la población de Irpinia desde la década de 1950, el terremoto de 1980 que causó 3.000 muertes y 280.000 personas desplazadas junto con un importante daño a la línea, además de las políticas de inversión en transporte por carretera, llevaron a la suspensión definitiva de la línea en 2010. Pero, por el fuerte valor histórico y el paisaje único que la linea atraviesa, desde 2007, la asociación Treni di Irpinia puso a disposición trenes turísticos para llegar a los pueblos durante eventos, destacando el potencial turistico de la línea.

En 2009, una red de asociaciones (Amici della Terra Irpina, Irpinia Turismo, RossoFisso, Irpinando), organizaciones y ciudadanos, junto con el Observatorio CGIL, crearon la asociación In_Loco_Motivi, para la recuperación y mejora de la línea, manteniendo una importante tensión a través de la participación en eventos a escala nacional, como el Día Nacional de los Ferrocarriles Olvidados. En la propuesta de estructura estratégica contenida en el PTCP Avellino 2014, la línea ferroviaria aparece como una "ruta de movilidad sostenible de interés histórico, arquitectónico y paisajístico de valor territorial" y se incluye entre los proyectos y programas considerados prioritarios para la consecución de los objetivos del Plan. La acción prevista se refiere a la adaptación de la línea ferroviaria y su promoción como ruta de interés turístico, a través de un acuerdo entre la Provincia, la Región, la Autoridad Ferroviaria, los municipios, las comunidades y los operadores económicos. En 2015, gracias a un acuerdo interistitucional, la línea se incluye en el proyecto Binari senza tempo, también gracias a la estrecha relación con la Ciclovia dell'acqua que conecta Caposele con Santa Maria di Leuca, parte del Sistema Nacional de Cicloturismo Turístico (MIT y Mibact 2015). Estas actividades le dieron a la línea una nueva identidad e importancia territorial, y gracias a estas iniciativas y al compromiso de las asociaciones involucradas, la línea se incluyó en la lista de la ley 128/2017. Hasta la fecha, se han restaurado los $120 \mathrm{~km}$ de línea y, a lo largo de las vías, circulan trenes turísticos. Un proyecto hecho posible por la sinergia entre las autoridades locales, la Fundación FS y la región de Campania. La línea Avellino-Rocchetta San Antonio representa un caso emblemático, debido a la fragilidad multisectorial del territorio que atraviesa, pero que hoy está buscando un cambio radical, convirtiéndose en el líder de nuevas prácticas de reutilización y participación, visibles en el papel de Alta Irpinia en el SNAI (Alta Irpinia es la única de las 68 áreas piloto seleccionadas para incluir un Plan de Transporte de Áreas Internas) y por la coherencia interinstitucional que este proyecto ha visto converger (Contarino, Von Normann, 2018) a partir de las comunidades y asociaciones de ciudadanos. Un hilo conductor que une proyectos de movilidad sostenible, leyes nacionales sobre ferrocarriles turísticos, planificación de grandes áreas, en un marco de coherencia que hizo posible que esta infraestructura desempeñara un papel portador de nuevas economías y valores.

\section{Experiencias de reutilización de líneas abandonadas}

\subsection{La Vía Verde dei Trabocchi}

Es posible caracterizar en una perspectiva "ecológica" numerosas experiencias de reutilización de las líneas ferroviarias que surgen en zonas de fragilidad e di valor medioambiental. En Italia, una experiencia emblemática en curso es la Vía Verde de la Costa dei Trabocchi, una ruta de 40 kilómetros a lo largo de la costa adriática de Abruzzo que resulta de la reutilización de la línea de ferrocarril Ancona-Lecce en el tramo Ortona-Vasto y que ha sido reconocida como una oportunidad estratégica para la regeneración de la franja costera. El proyecto surge del desmantelamiento de la línea ferroviaria que entró en servicio en 1864 y fue abandonada en fases diferentes, entre 1985 y 2005, tras la construcción de una variante de trazado, construida 


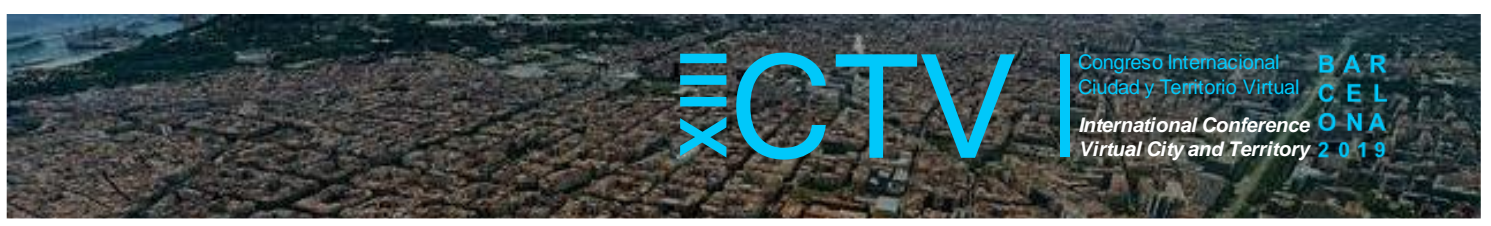

más internamente, a causa de la fragilidad medioambiental que implicaba altos costos de gestión, dificultad en la rehabilitación y potencial de valorización costera (Amato et al., 2019).

El proyecto de la green way tiene sus raíces en el Proyecto territorial especial de la Costa teatina, previsto por el Plan Territorial General Provincial de Chieti (2002), en conformidad con el Quadro di Riferimento Regionale (2000) y con el art.41 Misure di tutela ambientale connesse alla dismissione di tratte ferroviarie de la Ley Regional 38/96 (modificado con la Ley Regional $5 / 07$ ) que garantiza la protección de las áreas alrededor del antiguo ferrocarril, originalmente imaginado como un posible ferrocarril turístico.

Figura 3. La Via Verde dei Trabocchi e la rete Bicitalia

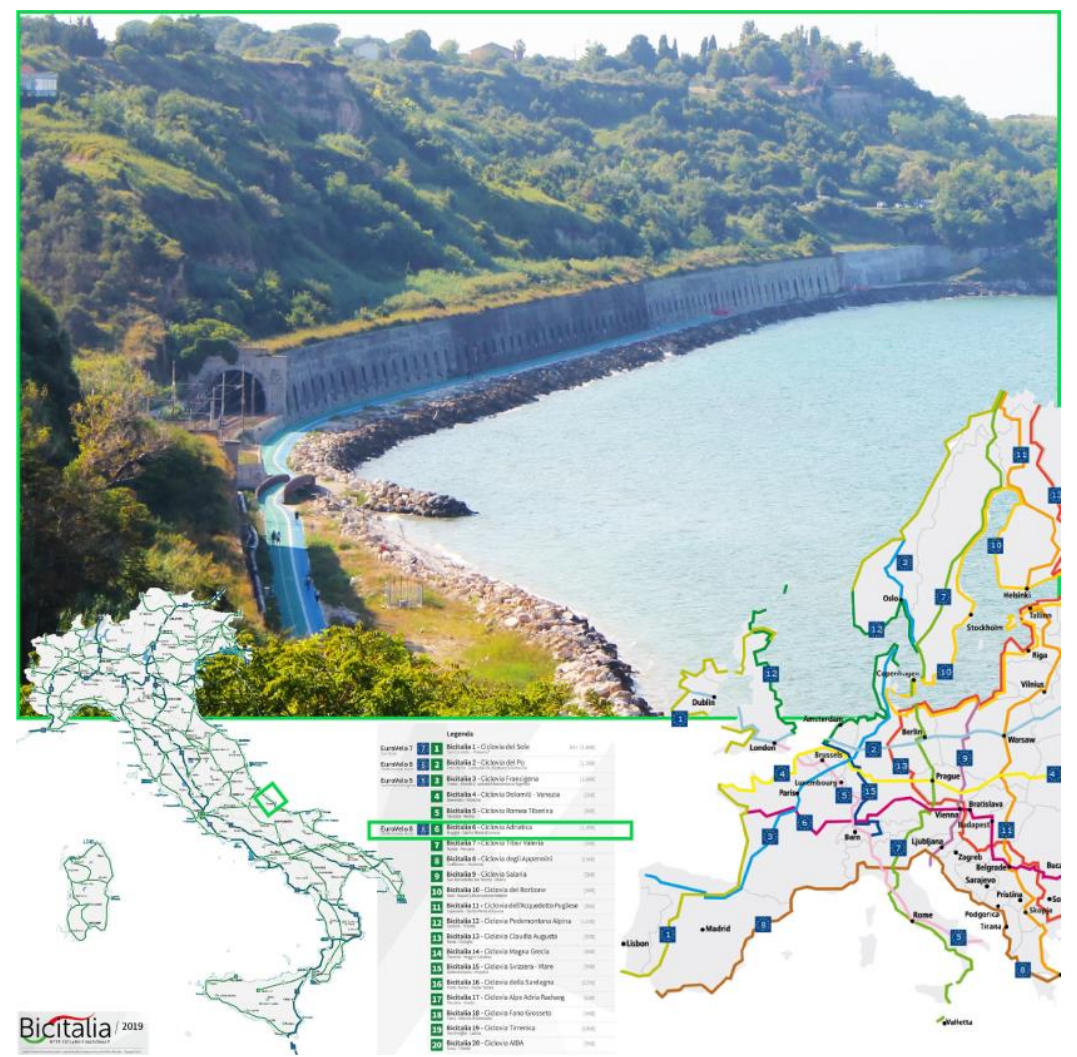

Fuente: C. Amato, 2019. Foto: Chiara Ravagnan.

La Ley Regional n. 5/07 identifica en este ambito territorial un "Sistema de Áreas Protegidas de la Costa Teatina" y configura la realización de la Vía Verde como un proyecto estructurante. El Proyecto preliminar de la green way se aprobó en 2009, llegando a ser parte del Documento programmatico del Progetto territoriale speciale della fascia costiera (2010) y del Protocollo di intesa per la realizzazione della Via Verde della Costa dei Trabocchi, estipulado entre RFI, Ministerios, Región, Provincia, Municipios (y otros actores) en 2011. El Protocollo sanciona la disponibilidad de RFI para la cesión de las áreas a título oneroso y el financiamiento con el Fondo Aree Sottoutilizzate (hoy FSC) por el Ministero dello Sviluppo economico, sentando las bases el proyecto ejecutivo de la "Vía verde della Costa dei Trabocchi" (2017), ahora en ejecución. La construcción de la ruta es parte del proceso de construcción de la Ciclovía Adriática que, en el marco de las 10 Ciclovie turistiche nazionali, se planifica desde Venezia hasta el Gargano a lo largo de $700 \mathrm{~km}$ de franja costera. El camino coincide con la rama o 6 


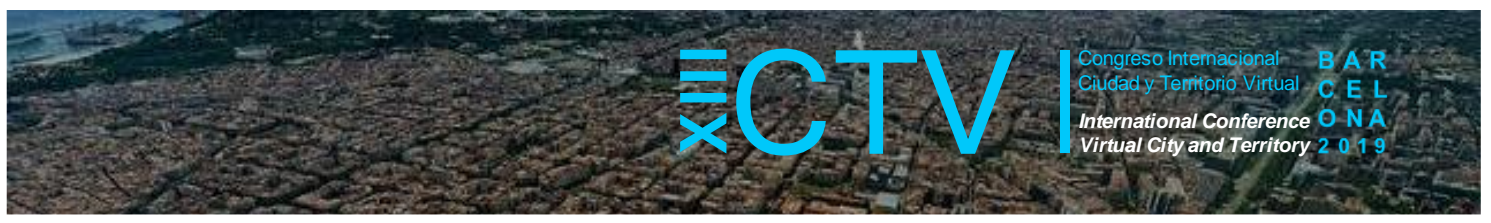

de la red nacional Bicltalia - en parte en la fase de diseño y en parte ya implementada - que se conecta con la red EuroVelo. En mayo de 2019, se firmó un Protocollo di intesa para el diseño y la construcción de la ruta ciclista turística del Adriático, entre el Ministero delle Infrastrutture e dei Trasporti y las Regiones de Marche (líder), Véneto, Emilia-Romagna, Molise, Puglia y Abruzzo. Para la finalización de esta ruta hay una primera asignación presupuestaria de 19 millones de euros, y la sección de Abruzzo ha sido confirmada como la más avanzada en la construcción de la Ruta Adriática: 86 km a lo largo de $131 \mathrm{~km}$. Además de insertar las Ciclovia en una política de movilidad sostenible, y de promover el uso de bicicletas en general, con la red de rutas ciclistas turísticas, el MIT tiene la intención de contribuir al desarrollo del ciclismo como impulso a la regeneración de los territorios atravesados. Las intervenciones dirigidas a la reutilización de la línea ferroviaria también han desencadenado acciones para restaurar y proteger los acantilados sujetos a una fuerte erosión y sifonamiento, regeneración del hábitat de las plantas autóctonas, contrarrestando los fenómenos de deslizamientos de tierra y la degradación ambiental.

La green way también soporta la "accesibilidad universal" de un bien común como la costa de Abruzzo, desarrollándose entre el mar y la colina que limita el acceso a la playa y a los landmarks diseminados en un paisaje de inestabilidad hidrogeológica. De hecho, la costa está salpicada de componentes de valor histórico y de identidad que enriquecen un sistema de promontorios. Entre otros, los Trabocchi, iconos de la fragilidad de la costa y, al mismo tiempo, elemento de continuidad para un posible renacimiento de este territorio a partir de una reconfiguración de las relaciones funcionales, morfológicas y ecológicas (Amato et al., 2019).

\subsection{La Via verde del Mar}

En la costa valenciana hay un caso emblemático comparable al que se está desarrollando a lo largo de la costa teatina, en el marco de un contexto nacional y regional fuertemente encaminado a incorporar las directrices medioambientales en términos de infraestructuras verdes: la Vía Verde del Mar. Una oportunidad para la regeneración territorial, en algunos casos de gran valor ambiental y paisajístico, que se desencadena por la disposición gradual y generalizada de las redes ferroviarias de vía estrecha a lo largo de las zonas costeras. El progreso tecnológico, junto con la mayor conciencia de la calidad y la fragilidad de los contextos ambientales, fue de hecho la causa del proceso masivo de eliminación que afectó a este tipo de línea ferroviaria a partir de la segunda mitad del siglo XX, para eliminar los medios de transporte lentos y obsoletos, con consecuencias negativas para las economías y las comunidades locales que estos conectaban (Ravagnan, 2019).

La Comunidad Autónoma Valenciana es un caso de estudio de particular interés. De particular interés ambiental y paisajístico es la Vía verde del Mar, en el municipio de Oropesa del Mar, en la Provincia de Castellón. Como en el caso de la Vía Verde dei Trabocchi, la ruta discurre entre una zona montañosa, la Sierra de Oropesa, y el mar, utilizando algunas "obras titánicas" creadas para el paso del ferrocarril, que hoy constituyen componentes de identidad: pasajes en trincheras, túneles, puentes metálicos. El ferrocarril, que forma parte de la ruta BarcelonaValencia, se construyó a finales del siglo XIX en una fase sin sensibilidad al contexto de alto valor natural y paisajístico. La variante de trazado, requerida por la necesidad de aumentar la velocidad en este tramo, llevó a la construcción de la Vía Verde del Mar en 2008-2009 impulsada por el Ministerio de Fomento, como compensación ambiental por la construcción de la variante. La reutilización de estas vías ferroviarias, que, en las primeras décadas, tuvo lugar 


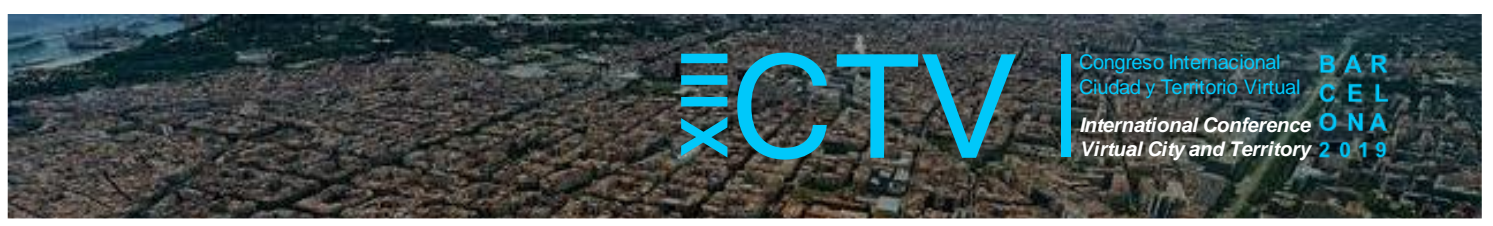

solo por tramos, ahora encuentra un enfoque de planificación territorial de gran interés en la construcción de una Infraestructura verde objeto del Plan de Acción Territorial de la Infraestructura Verde del Litoral (PATIVEL). EI PATIVEL es un instrumento de planificación del territorio supramunicipal, al que se refiere el artículo 16 de la Ley 5/2014 (LOTUP) de la Generalitat, relativa al Gobierno del territorio, la Planificación Urbana y el Paisaje, que puede adoptar un carácter sectorial o integrado, basado en las peculiaridades temáticas y el área territorial identificada. En el caso del PATIVEL, se explica una dimensión territorial que traduce una instancia de integración entre los aspectos urbanos, ambientales y paisajísticos en la construcción de una infraestructura costera verde. Este instrumento adquiere el carácter de plan territorial con el objetivo de superar un enfoque puramente cuantitativo y de protección, a fin de preservar unas continuidades paisajisticas y infraestructurales estratégicas, aunque menos valiosas que las áreas naturales protegidas; al mismo tiempo, sugerir oportunidades de desarrollo socioeconómico para la zona costera, en línea con la Estrategia Territorial de la Comunidad Valenciana (Decreto 1/2011). En particular, el PATIVEL asocia objetivos para reducir el consumo de suelo con medidas de mejora del paisaje. En el caso de la Vía verde del Mar, el PATIVEL prevé no solo la conexión en red del tramo de la via verde con un corredor costero ciclista peatonal, sino que prescribe la no construcción de las áreas circundantes, preservando la conexión ecológica y la continuidad del paisaje entre la colina y el mar.

\section{Figura 4. La Via Verde del Mar, Vias Verdes y Eurovelo}

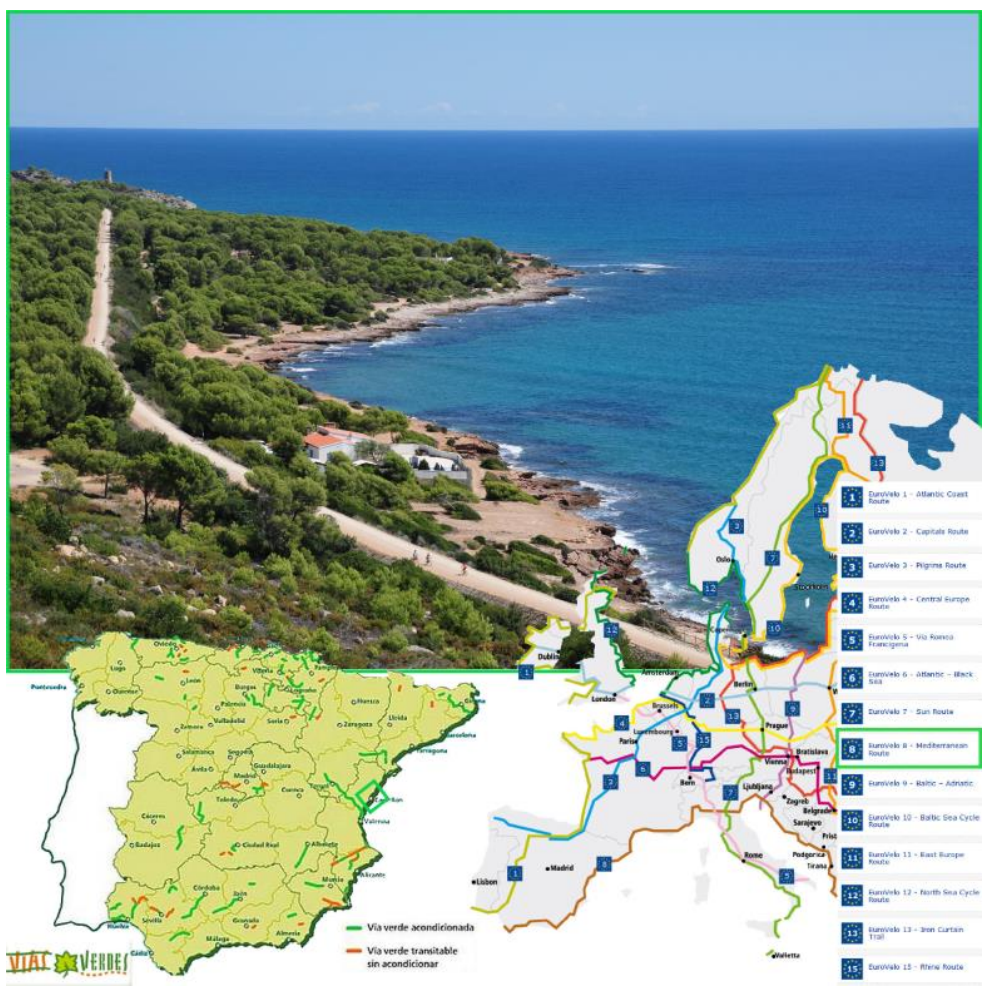

Fuente: C. Amato, 2019.

\section{Conclusiones. Perspectivas y nuevas referencias para la experimentación}

El analisis de los estudios de caso resalta las diferentes connotaciones de fragilidad de los territorios investigados, en las que surgen los problemas relacionados con los procesos de desmantelamiento de las líneas ferroviarias: pérdida de accesibilidad, vaciado de zonas de 


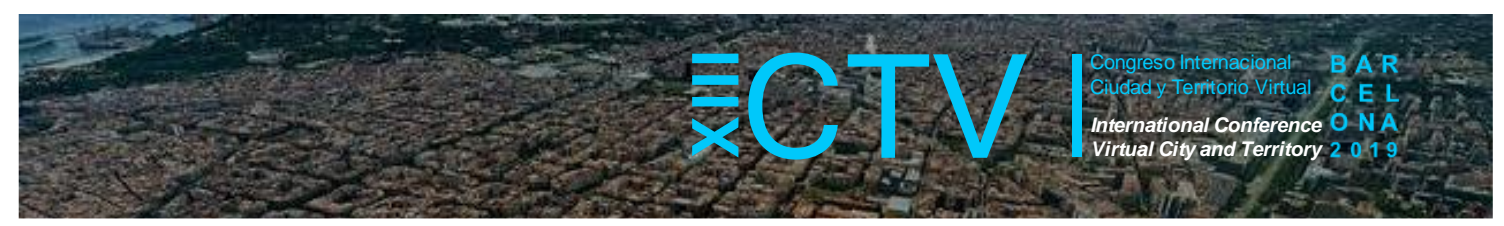

influencia, inseguridad e insuficiencia de los trazados. En particular, los casos investigados responden a algunos problemas específicos:

- Eliminación de líneas menores en áreas caracterizadas por la marginalidad (montaña, transfronteriza), cuya reactivación requiere estrategias para adaptarse a los cambios socioeconomicos, garantizando la habitabilidad del territorio a través de formas integradas, multinivel y asociativas de gestion de las redes infraestructurales y de los servicios;

- Desmantelamiento de líneas en áreas históricamente rurales o deshabitadas de gran extensión, que por su valor paisajístico e histórico sugieran acciones relacionadas con la mejora del paisaje en sinergia con la mejora de los activos de identidad comunes, a través de nuevas políticas "ad hoc" y nuevas formas de gestión del patrimonio, también temporales y participativos, que desarrollan nuevas economías para los "espacios a no ser consumidos", haciendo "red" entre los sujetos activos en el territorio;

- Desmantelamiento resultado de variación de trazado en áreas de alta sensibilidad y naturalidad que requieren nuevas formas de movilidad sostenible, en línea con medidas de seguridad y restricciones de protección ambiental que exceden la fragilidad ambiental.

Los fenómenos descritos confirman la oportunidad de poner en juego diferentes perspectivas disciplinarias y nuevas políticas relacionadas con las infraestructuras, que sean promotores de:

a) nuevas categorías de proyectos integrados e interescalares para la planificación de redes de infraestructura en relación con las tres perspectivas y la fragilidad de los territorios, que se convierten en insumos de diseño para la resiliencia: corredores multimodales, ferrocarriles turísticos, vías verdes, que son portadores de estrategias de regeneración dirigidas a relanzar el servicio, adaptar las líneas para el desarrollo turístico, reutilizarlas de manera sostenible, vinculando el replanteamiento de las infraestructuras a los nuevos escenarios territoriales;

b) nuevos criterios operativos que recuerdan una instancia de innovación en la dimensión estructural-estratégica de la planificación, capaces de mejorar los diseños como "cifras de reestructuración" que ponen en sinergia instrumentos ordinarios y extraordinarios, recursos públicos y privados, escenarios a largo y corto plazo;

c) nuevas formas de gestión de servicios y activos ferroviarios conectados a partenariado, hileras y redes institucionales, a diferentes niveles nacionales y europeos.

En esta dirección, la experimentación muestra un esfuerzo por integrar las intervenciones conectadas a las fragilidades, en un intento de enfrentar las relaciones entre los diferentes aspectos para definir estrategias efectivas para revertir los procesos de crisis, abandono y despoblación a partir de una sinergia entre transformaciones físico-funcionales, modelos de movilidad, protección y mejora ambiental y paisajística. Al mismo tiempo, parece fundamental poner en sintonía las innovaciones derivadas de las políticas y programas nacionales, europeos y regionales a través de procedimientos dirigidos a la gobernanza multinivel y la cooperación interinstitucional, e integrar recursos y energías provenientes de comunidades, operadores, administraciones locales y municipios que participan activamente en estos procesos.

En este sentido, el camino de investigación pretende profundizar, a lo largo de la segunda fase actualmente en curso, los aspectos relacionados con la viabilidad económica de los diversos escenarios de intervención y llegar, en la fase final, a la definición de directrices para el relanzamiento y reutilización de líneas ferroviarias en territorios frágiles. 


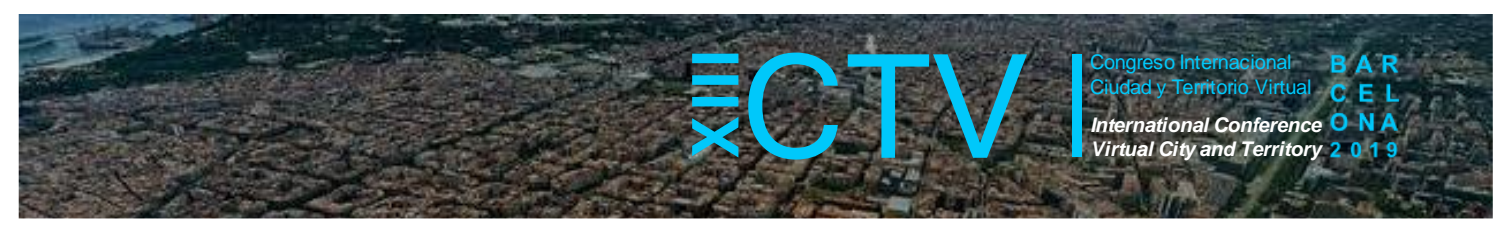

Agradecimientos: Proyecto de investigación "Percorsi di resilienza. II rilancio e il riuso delle linee ferroviarie minori nei territori fragili" financiado por Sapienza Università di Roma.

Contribuciones de los autores: C. Amato ha desarrollado los $\S 1$ y 4.1 ; C. Ravagnan los $\S 2$ y 5.1, F. Rossi los $\S 4.2$ y 5.2. El $\S 3$ ha sido elaborado por C. Amato y J. M. Ureña y el $\S 6$ ha sido desarrollado por C. Ravagnan y J. M. Ureña.

Conflicto de Intereses: Los autores declaran que no hay conflicto de intereses.

\section{Bibliografia}

Amato, C.; Ravagnan, C. y Rossi, F. (2019). Between legacy and abandonment. The reuse of minor railways as resilience paths. En C. Gambardella. (Ed.), World Heritage and Legacy, Culture, Creativity, Contamination. En Proceedings of the XVII International Forum Le Vie dei Mercanti (p. 217-226). Roma, Italia: Gangemi.

Amato, C. (2018). II potenziale della rete ferroviaria secondaria per il rilancio dei territori fragili italiani. En G. Fini, V. Saiu, C. Trillo. (Eds.), UPhD Green. Il dottorato come luogo esplorativo della ricerca sulla sostenibilità. Planum Magazine, II (37), 7-14.

Carta, M. y Ronsivalle, D. (2015). Territori interni. La pianificazione integrata per lo sviluppo circolare: metodologie, approcci, applicazioni per i nuovi cicli di vita. Roma, Italia: Aracne

Cerasoli, M. (2015). Qualità urbana, mobilità, qualità della vita: una "grammatica" per il Rinascimento della città, Urbanistica Informazioni, 07(263), 16-19.

Cerasoli, M. y Mattarocci, G. (2018). Rigenerazione urbana e mercato immobiliare. Roma, Italia: Ro-maTrE-Press.

AAVV (2017). PROFECY - Processes, Features and Cycles of Inner Peripheries in Europe. Final Report. Recuperado de https://www.espon.eu/inner-peripheries

Del Molino, S. (2016). La España vacía. Viaje per un pais que nunca fue. Madrid, España: Turner.

Ruiz de la Riva E. et al. (2015). Territorio y paisaje: notas metodológicas en torno al Plan Especial de Protección de los Valles del Nansa (Cantabria, España). Ciudad y territorio: Estudios territoriales, 184, 1133-4762.

de Ureña J. M., ed (2012), Territorial Implications of High Speed Rail: A Spanish perspective, Farnham, UK: Ed. Ashgate.

Dematteis, G. (2001). Introduzione. Tema, articolazione e risultati della ricerca. En G. Dematteis y F. GOVERNA. (Eds.), Contesti locali e grandi infrastrutture. Politiche e progetti in Italia e in Europa (pp.11-23). Milano, Italia: FrancoAngeli.

Di Venosa, M. et al. (2018). Rigenerare territori fragili. Strategie e progetti. Roma, Italia: Aracne.

Hirschman, A. O. (1958). The strategy of economic development. New Haven, Conn.: Yale University Press.

Ravagnan, C. (2019). Rigenerare le città e i territori contemporanei. Prospettive e nuovi riferimenti operativi per la sperimentazione, Roma: Aracne.

Ricci, L. (2018). Costruire lo spazio pubblico, tra storia, cultura e natura. En L. Ricci; A. Battisti; V. Cristallo y C. Ravagnan. (Eds.), Costruire la città pubblica. Tra storia, cultura e natura (pp. 11-17). Recuperado de http://www.urbanisticainformazioni.it/-015-92-.html 